\title{
Remark on the Energy Spectrum of a Decaying System
}

\author{
Pavel Exner \\ Department of Theoretical Nuclear Physics, Faculty of Mathematics and Physics of the \\ Charles University, 11000 Praha 1, Czechoslovakia
}

\begin{abstract}
Some time-evolution operators of a general unstable system lead to unphysical spectrum (unbounded below) of the total Hamiltonian. Various necessary conditions for boundeness of the spectrum are known. It is shown here, how this spectrum can be determined, which, in particular, gives the sufficient condition.
\end{abstract}

\section{Introduction}

It is well-known, that the exponential decay law, though is confirmed experimentally in a wide range, has an unphysical property; it corresponds to the energy spectrum without (lower) bound. Also some similarly behaving decay laws in the general scheme for description of unstable systems (developed mostly in the last few years - see e.g. Refs. [1-5]) exhibit this unpleasant feature. Various authors [3-7] deduce different conditions for the "reduced evolution operator" (time-evolution operator of the unstable system itself), under which spectrum of the total Hamiltonian is (below) unbounded. Nevertheless, the same question arises for those reduced evolution operators which do not obey any of the mentioned conditions. We shall show here how the spectrum can be determined to a given reduced evolution operator. It gives, in particular, a possibility to decide whether a reduced evolution operator corresponds to a total Hamiltonian with a spectrum bounded below.

In Section 2 we collect the assumptions in our description of unstable systems and introduce some notions. Section 3 is devoted to derivation of relations between a reduced evolution operator and spectrum of the corresponding total Hamiltonian. A simple criterion is given for the case when the state Hilbert space of an unstable system is finite-dimensional. The last section contains discussion of the results, a special attention being payed to connections of decay laws to the total Hamiltonian. 


\section{Preliminaries}

We deal with the description of an unstable system considered e.g. in Ref. [2]. Let us remind that such a system is assumed to obey the following conditions:

(i) one can ascribe to it a state Hilbert space $\mathscr{H}_{u}$,

(ii) $\mathscr{H}_{u}$ is a proper subspace of a Hilbert space $\mathscr{H}$,

(iii) a strongly continuous unitary representation $U(t)$ of one-parameter group of translations is realized on $\mathscr{H}$ and $\mathscr{H}_{u}$ is not an invariant subspace of $U(t)$ on $\mathscr{H}$ for any $t>0$.

Here $\mathscr{H}$ is understood to be state Hilbert space of the "whole" system, i.e. including decay products as well. Further $U(t)=\exp (-i H t)$ plays role of the time evolution operator of the considered system, and $H$ is therefore the total Hamiltonian.

Time evolution on the space $\mathscr{H}_{u}$ itself is governed by the reduced evolution operator

$$
V(t)=E_{u} U(t) E_{u}, \quad t \in \mathbb{R},
$$

where $E_{u}$ is a projection, $E_{u} \mathscr{H}=\mathscr{H}_{u}$. The relations

$$
\begin{aligned}
& V(t)=V^{+}(-t), \\
& \|V(t)\| \leqq 1
\end{aligned}
$$

hold obviously for all real $t$. The reduced evolution operator determines the decay law: assuming the unstable system to be prepared at $t=0$ in a (normalized) state $\psi \in \mathscr{H}_{w}$, we define it as

$$
P_{\psi}(t)=\|V(t) \psi\|^{2},
$$

in particular

$$
P_{\psi}(t)=|(\psi, V(t) \psi)|^{2}
$$

if $\operatorname{dim} \mathscr{H}_{u}=1$. More generally if the system is initially prepared in a mixed state described by density matrix $\varrho$, Ran $\varrho \subset \mathscr{H}_{w}$, we define (cf. Ref. [8]):

$$
P_{\varrho}(t)=\operatorname{Tr}\left\{V^{+}(t) V(t) \varrho\right\} .
$$

We are interested in a class of operator-valued functions which are allowed to play role of reduced evolution operators. Solution of this problem is contained in the theory of unitary dilations (this theory was firstly applied to description of unstable systems by Williams [3]); let us remind it here briefly.

Following Sz.-Nagy and Foias [9] we introduce the concept of positive definite operator-valued function ${ }^{1}$ (PO-function).

1 We formulate the statements of Sz.-Nagy and Foias with respect to purposes of this paper. The notion of PO-function as well as the assertion of the following theorem are in [9] considered for more general functions $V: G \rightarrow \mathscr{L}(\mathscr{H}), G$ being a (topological) group 
Definition 1. A function $V: \mathbb{R} \rightarrow \mathscr{L}(\mathscr{H})$ (whose values are bounded operators on a Hilbert space $\mathscr{H}$ ) is called positive definite if the inequality

$$
\sum_{i, j=1}^{n}\left(\psi_{i}, V\left(t_{i}-t_{j}\right) \psi_{j}\right) \geqq 0
$$

holds for any integer $n$ and arbitrary $\psi_{1}, \ldots, \psi_{n} \in \mathscr{H} ; t_{1}, \ldots, t_{n} \in \mathbb{R}^{2}$

Remark. If $\operatorname{dim} \mathscr{H}=1$, the present definition coincides with the usual definition of positive definite function $V: \mathbb{R} \rightarrow \mathbb{C}-$ cf. Ref. [10]. The condition (2.4) is then expressed as follows: for any integer $n$ and arbitrary $\alpha_{1}, \ldots, \alpha_{n} \in \mathbb{C} ; t_{1}, \ldots, t_{n} \in \mathbb{R}$, the inequality

$$
\sum_{i, j=1}^{n} \bar{\alpha}_{i} \alpha_{j} V\left(t_{i}-t_{j}\right) \geqq 0
$$

holds.

We are interested especially in those PO-functions which are weakly continuous (WPO-functions). Let us now formulate for our purposes the following theorem originally deduced by Sz.-Nagy [11]:

Theorem 1. (i) Let $U: \mathbb{R} \rightarrow \mathscr{L}(\mathscr{H})$ be a unitary representation of one-parameter group of translations on $\mathscr{H}$, and let $\mathscr{H}_{u}$ be a subspace of $\mathscr{H}$. Then the function $V: V(t)=E_{u} U(t) E_{u}$ is $P O$-function and obeys $V(0)=E_{u}$. Moreover, if $U$ is continuous (weakly or strongly, what is the same due to unitarity), then $V$ is also continuous.

(ii) Conversely, to any PO-function $V: \mathbb{R} \rightarrow \mathscr{L}\left(\mathscr{H}_{u}\right), V(0)=E_{u}$, there exists a unitary representation $U: \mathbb{R} \rightarrow \mathscr{L}(\mathscr{H})$ ("the minimal unitary dilation") of oneparameter translation group on some Hilbert space $\mathscr{H}, \mathscr{H} \supset \mathscr{H}_{u}$, so that it holds

$$
V(t)=E_{u} U(t) E_{u}
$$

for all $t$ and the space $\mathscr{H}$ obeys the minimality condition

$$
\mathscr{H}=\overline{\left.\bigcup_{t \in \mathbb{R}} U(t) \mathscr{H}_{u}\right]_{i}} \cdot{ }^{3}
$$

Moreover, if $V$ is weakly continuous, then the representation $U$ is (weakly, and therefore also strongly) continuous.

Corollary. The function $V: \mathbb{R} \rightarrow \mathscr{L}\left(\mathscr{H}_{u}\right)$ can be a reduced evolution operator on $\mathscr{H}_{u}$ if and only if it is WPO-function such that $V(0)=E_{u}$.

This class of reduced evolution operators is, however, too large. Some of them can be excluded, because they violate physical assumptions, which are to any system (especially to an unstable one) naturally ascribed. As we have mentioned, a reason for such exclusion can consist of the fact that the Hamiltonian $H$ (generator of the minimal unitary dilation) corresponding to given $V(t)$ has a spectrum (below) unbounded.

Let us show some reduced evolution operators with this unpleasant property:

2 Notice that the relation (2.4) implies $|(\psi, V(t) \psi)| \leqq(\psi, V(0) \psi), V(0)$ being Hermitian and positive, and $V^{+}(\mathrm{t})=V(-t)$; these conditions are closely connected to (2.2)

3 The symbol $M_{\lambda}$ means the linear envelope of a set $M$, i.e. the collection of all finite linear combinations of vectors belonging to $M$ 
Theorem 2. (i) Let $\{V(t)\}_{t \geqq 0}$ be a continuous semigroup of contractions, i.e.

$$
V\left(t_{1}\right) V\left(t_{2}\right)=V\left(t_{1}+t_{2}\right), \quad t_{1}, t_{2} \geqq 0 .
$$

Equivalently, let any regeneration of a decayed state be forbidden:

$$
E_{u} U\left(t_{1}\right)\left(I-E_{u}\right) U\left(t_{2}\right) E_{u}=0, \quad t_{1}, t_{2} \geqq 0 .
$$

(ii) Let a regeneration be forbidden after a finite time $T_{r}$ :

$$
V\left(t_{1}\right) V\left(t_{2}\right)=V\left(t_{1}+t_{2}\right), t_{1} \geqq T_{r}>0, t_{2} \geqq 0 .
$$

(iii) Let $V(t)$ be weakly bounded by an exponential, i.e. let $\psi, \chi \in \mathscr{H}_{u}$ and $\gamma>0$ exist so that

$$
|(\psi, V(t) \chi)| \leqq \exp (-\gamma t), \quad t \geqq 0
$$

(iv) For $\operatorname{dim} \mathscr{H}_{u}=1$ let the integral

$$
\int_{-\infty}^{\infty} \frac{\ln |(\psi, V(t) \psi)|}{1+t^{2}} d t
$$

diverge.

Any of the conditions (i)-(iv) implies that the spectrum $\sigma(H)$ is (below) unbounded. Moreover, the validity of (i) or (ii) implies $\sigma(H)$ to be the whole real line.

For proof of this theorem see for example [3] - (i), [4] - (i), [5] - (i), (ii), [6] - (iii), [7] - (iv). Negation of each of conditions (i) (iv) is necessary conditions for $V(t)$ to correspond to a (below) bounded spectrum $\sigma(H)$. In the next section we shall show a sufficient condition.

\section{The Spectrum of Minimal Unitary Dilation}

The first thing which we need is some general expression of WPO-functions. Let us start with the Bochner-Khintchin theorem:

Theorem 3. To any continuous positive definite function $V: \mathbb{R} \rightarrow \mathbb{C}$ (see Remark to Definition 1) there exists a bounded real function $\omega$, non-decreasing and continuous on the right, so that

$$
V(t)=\int_{-\infty}^{\infty} e^{-i \lambda t} d \omega(\lambda), \quad t \in \mathbb{R}
$$

Conversely, any such function $\omega$ defines through (3.1) a continuous positive definite function $V$. 
For proof see e.g. Ref. [10]. Notice further that the function $\omega$ (which determines a Lebesgue-Stieltjes measure on $\mathbb{R}$ ) can be found to given $V(t)$ by means of the formulae (cf. Ref. [10])

$$
\begin{aligned}
& \varphi(\xi+i \eta)=\int_{0}^{\infty} V(t) e^{i t(\xi+i \eta)} d t, \quad \eta>0 \\
& \frac{1}{2}\left(\omega_{0}(\lambda-0)+\omega_{0}(\lambda+0)\right)=\frac{1}{\pi} \lim _{\eta \rightarrow 0+} \int_{0}^{\lambda} \operatorname{Re} \varphi(\xi+i \eta) d \xi \\
& \omega(\lambda)=-\lim _{\lambda \rightarrow-\infty} \omega_{0}(\lambda)+\omega_{0}(\lambda+0) .
\end{aligned}
$$

In particular, if $\omega$ is absolutely continuous, i.e. if $\omega(\lambda)=\int_{-\infty}^{\lambda} f(\lambda) d \lambda, f \in L(\mathbb{R})$, the formulae (3.2) reduce themselves to the inverse Fourier transformation. We shall generalize now Theorem 3 for the case of WPO-functions:

Theorem 3a. An operator-valued function $V: \mathbb{R} \rightarrow \mathscr{L}\left(\mathscr{H}_{u}\right)$ is weakly continuous and positive definite if and only if there exists an operator valued function $F: \mathbb{R} \rightarrow \mathscr{L}\left(\mathscr{H}_{u}\right)$ such that

(a) for all $\lambda \in \mathbb{R}$ the operator $F(\lambda)$ is Hermitian,

(b) for all $\lambda, \mu \in \mathbb{R}, \lambda \leqq \mu$, it holds $F(\lambda) \leqq F(\mu) \leqq F(\infty), F(\infty)$ being a Hermitian operator on $\mathscr{H}_{u}$,

(c) $F$ is weakly continuous on the right, i.e. for all $\psi, \varphi \in \mathscr{H}_{u}$ it holds $(\psi, F(\lambda+0) \varphi)$ $=(\psi, F(\lambda) \varphi)$,

(d) $F(\lambda)$ is related to $V(t)$ by means of the equation

$$
V(t)=\int_{-\infty}^{\infty} e^{-i \lambda t} d F(\lambda)
$$

Remark. The formula (3.3) has to be read as follows: to a given $t$ and arbitrary $\psi, \varphi \in \mathscr{H}_{u}$ the equality holds

$$
(\psi, V(t) \varphi)=\int_{-\infty}^{\infty} e^{-i \lambda t} d(\psi, F(\lambda) \varphi)
$$

Other formulae containing integrals of the type (3.3) are understood in the same sense.

Proof.. Sufficient Condition. Let a function $F$ obeying $(a-d)$ be given. Let us choose an integer $n$ and some (arbitrary) $t_{1}, \ldots, t_{n} \in \mathbb{R} ; \psi_{1}, \ldots, \psi_{n} \in \mathscr{H}_{u}$. Then we obtain

$$
\begin{aligned}
& \sum_{i, j=1}^{n}\left(\psi_{i}, V\left(t_{i}-t_{j}\right) \psi_{j}\right)=\sum_{i, j=1}^{n} \int_{-\infty}^{\infty} e^{-i \lambda\left(t_{i}-t_{j}\right)} d\left(\psi_{i}, F(\lambda) \psi_{j}\right) \\
& =\int_{-\infty}^{\infty} d\left(\sum_{i=1}^{n} e^{i \lambda t_{i}} \psi_{i}, F(\lambda) \sum_{j=1}^{n} e^{i \lambda t_{j}} \psi_{j}\right) \geqq 0
\end{aligned}
$$

4 The function $\omega$ defined by (3.2c) obeys the condition $\lim \omega(\lambda)=0$; under this condition the correspondence $V \leftrightarrow \omega$ is one-to-one 
so that $V$ is positive definite. Further for any $\psi \in \mathscr{H}_{u}$ the function $(\psi, V(t) \psi)$ as the Fourier transform of a finite Lebesgue-Stieltjes measure [see (3.3a)] is continuous. The polarization identity (cf. e.g. [12]) implies now the weak continuity.

Necessary Condition. For any $\psi \in \mathscr{H}_{u}$ the function $(\psi, V(t) \psi)$ is positive definite so that according to Theorem 3 a function $\omega_{\psi}$ exists:

$$
(\psi, V(t) \psi)=\int_{-\infty}^{\infty} e^{-i \lambda t} d \omega_{\psi}(\lambda) .
$$

Let us define for any $\lambda \in \mathbb{R}$ the operator $F(\lambda)$ in the following way:

$$
\begin{aligned}
(\psi, F(\lambda) \varphi) \equiv & \frac{1}{4}\left\{\omega_{\psi+\varphi}(\lambda)-\omega_{\psi-\varphi}(\lambda)-i \omega_{\psi+i \varphi}(\lambda)\right. \\
& \left.+i \omega_{\psi-i \varphi}(\lambda)\right\} .
\end{aligned}
$$

Since $V(0)$ is a bounded operator, the operator $F(\lambda)$ is obviously bounded; it assures that its definition through "matrix elements" is consistent. One easily verifies $(a-c)$ with the use of properties of the functions $\omega_{\psi}(\lambda)=(\psi, F(\lambda) \psi)$. Validity of the Equation (3.3) follows directly from the definition of the function $F(\lambda)$.

One can find the function $F(\lambda)$ corresponding to given WPO-function $V(t)$ by means of the formulae:

$$
\begin{aligned}
& \Phi(\xi+i \eta)=\int_{0}^{\infty} V(t) e^{i t(\xi+i \eta)} d t, \quad \eta>0, \\
& \frac{1}{2}\left[\Omega_{0}(\lambda-0)+\Omega_{0}(\lambda+0)\right] \\
& =\frac{1}{2 \pi} \lim _{\eta \rightarrow 0+} \int_{0}^{\lambda}\left[\Phi(\xi+i \eta)+\Phi^{+}(\xi+i \eta)\right] d \xi, \\
& F(\lambda)=-\underset{\lambda \rightarrow-\infty}{w-\lim _{0}} \Omega_{0}(\hat{\lambda})+\Omega_{0}(\lambda+0),
\end{aligned}
$$

which follow easily from (3.2), (3.4) (see Remark following Theorem 3a). The function $F(\lambda)$ constructed in this way corresponds uniquely to given $V(t)$, because due to $(3.5 \mathrm{c})$ it obeys the condition

$$
\underset{\lambda \rightarrow-\infty}{w-\lim _{\lambda \rightarrow-\infty}} F(\lambda)=0
$$

which removes the possible non-uniqueness $F(\lambda) \rightarrow F^{\prime}(\lambda)=F(\lambda)+F_{0}, F_{0}$ being any Hermitian ( $\lambda$-independent) operator.

Let $V(t)$ be now a reduced evolution operator, i.e. WPO-function such that $V(0)=E_{u}$. The minimal unitary dilation $U(t)$ of $V(t)$, as well as its generator $H$ we may write in the form of spectral representation

$$
U(t)=\int_{-\infty}^{\infty} e^{-i \lambda t} d E_{H}(\lambda), \quad H=\int_{-\infty}^{\infty} \lambda d E_{H}(\lambda)
$$

The function $E_{u} E_{H}(.) E_{u}$ fulfills the assumptions $(a-d)$ of Theorem $3 \mathrm{a}$ and, moreover, it obeys the condition (3.6). Thus (2.1), (3.3), and (3.7) imply

$$
F(\lambda)=E_{u} E_{H}(\lambda) E_{u}, \quad \lambda \in \mathbb{R},
$$


and

$\|F(\lambda)\| \leqq 1, \quad \lambda \in \mathbb{R}$.

Conventionally $\sigma(H)$ denotes the spectrum of $H$; further we shall introduce the following notion:

Definition 2. Let $V(t)$ be a reduced evolution operator. The set $\sigma[V] \equiv\{\lambda \in \mathbb{R} \mid$ $F(\lambda+\varepsilon)-F(\lambda-\varepsilon)>0$ for all $\varepsilon>0\}$ is called energy support of $V(t) .^{5}$

Now we are in position to prove our main theorem:

Theorem 4. Let $V(t)$ be a reduced evolution operator and $H$ be generator of the corresponding minimal unitary dilation. Then

$$
\sigma(H)=\sigma[V] .
$$

In particular, if $\sigma[V]$ is (below) bounded so is $\sigma(H)$.

Proof. A point $\lambda_{0}$ does not belong to $\sigma(H)$ if and only if there exists $\eta>0, E_{H}\left(\lambda_{0}+\eta\right)$ $-E_{H}\left(\lambda_{0}-\eta\right)=0-\mathrm{cf}$. [12]. Then (3.8a) implies $\lambda_{0} \notin \sigma[V]$, and therefore $\sigma[V] C$ $\sigma(H)$. On the other hand, let us consider the set

$$
\mathscr{M}=\bigcup_{t \in \mathbb{R}} U(t) \mathscr{H}_{u} .
$$

If $\lambda_{0} \notin \sigma[V]$, there exists $\eta>0$ such that $F\left(\lambda_{0}+\eta\right)-F\left(\lambda_{0}-\eta\right)=0$. Then the relation $(*) \quad\left(\varphi, E_{H}\left(\lambda_{0}+\eta\right) \varphi\right)-\left(\varphi, E_{H}\left(\lambda_{0}-\eta\right) \varphi\right)=0$

holds for any $\varphi \in \mathscr{M}$, because such expressions one can write as

$$
\left(\varphi, E_{H}(\lambda) \varphi\right)=\left(\psi, U^{+}(t) E_{H}(\lambda) U(t) \psi\right)
$$

for some $\psi \in \mathscr{H}_{u}$. Using the fact that $U(t)$ for any $t$ commutes with $E_{H}(\lambda)$ we obtain

$$
\left(\varphi, E_{H}(\lambda) \varphi\right)=\left(\psi, E_{H}(\lambda) \psi\right)=(\psi, F(\lambda) \psi) .
$$

Since $E_{H}(\Delta) \equiv E_{H}\left(\lambda_{0}+\eta\right)-E_{H}\left(\lambda_{0}-\eta\right)$ is a projection on $\mathscr{H}$, the relation $(*)$ implies

$$
E_{H}(\Delta) \varphi=0
$$

for all $\varphi \in \mathscr{M}$. Let us assume now $E_{H}(\Delta) \neq 0$, i.e. that there exists $\chi \in \mathscr{H},\left\|E_{H}(\Delta) \chi\right\|=$ $\varepsilon>0$. The minimality condition (2.5) states that the linear envelope $\mathscr{M}_{\lambda}$ of $\mathscr{M}$ is dense in $\mathscr{H}$, so that to given $\chi$ one can find a finite linear combination $\chi_{0}=\sum_{i=1}^{n} \alpha_{i} \varphi_{i}, \varphi_{i} \in \mathscr{M},\left\|\chi-\chi_{0}\right\|<\varepsilon$. Then it holds

$$
\varepsilon=\left\|E_{H}(\Delta) \chi\right\|=\left\|E_{H}(\Delta)\left(\chi-\chi_{0}\right)\right\| \leqq\left\|\chi-\chi_{0}\right\|<\varepsilon,
$$

what contradicts to the assumption $E_{H}(\Delta) \neq 0$. Thus we obtain $\lambda_{0} \notin \sigma(H)$, and consequently $\sigma(H) \subset \sigma[V]$.

5 Let us point out that the present definition is suitable also for complex continuous positive definite functions; we have then $\sigma[V] \equiv\{\lambda \in \mathbb{R} \mid \omega(\lambda+\varepsilon)-\omega(\lambda-\varepsilon)>0$ for all $\varepsilon>0\}$. 
Discussion of this result will be performed in the next section. Here we show a simple consequence:

Corollary. If $\operatorname{dim} \mathscr{H}_{u}<\infty$, the relation

$$
\sigma(H)=\sigma[v]
$$

holds, where $v(t)=\operatorname{Tr} V(t), t \in \mathbb{R}$. In particular, if $\sigma[v]$ is (below) bounded so is $\sigma(H)$.

Proof. The finite dimension of $\mathscr{H}_{u}$ assures that the functions $v(t)$ and $\operatorname{Tr} F(\lambda)$ are defined. The function $v(t)$ as a finite sum of continuous positive definite functions is continuous and positive definite (cf. Theorem 3); one can express it as

$$
v(t)=\int_{-\infty}^{\infty} e^{-i \lambda t} d \operatorname{Tr} F(\lambda)
$$

There holds obviously $\sigma[v] \subset \sigma[V]$. On the other hand, all the functions $(\psi, F(\lambda) \psi)$ are non-decreasing, i.e. $\operatorname{Tr} F(\lambda+\varepsilon)-\operatorname{Tr} F(\lambda-\varepsilon)=0$ implies $F(\lambda+\varepsilon)-F(\lambda-\varepsilon)=0$, and consequently $\sigma[v] \supset \sigma[V]$.

\section{Discussion}

Theorem 4 together with the formulae (3.5) shows, how the spectrum of total Hamiltonian can be found to a given reduced evolution operator. Let us point out, that this procedure often simplifies:

(a) in the case of finite-dimensional $\mathscr{H}_{u}$ Corollary of Theorem 4 can be applied; its main advantage is that the all necessary informations are contained in one complex function only,

(b) under some reasonable assumptions about $V(t)$ (see e.g. [5] - Theorem 4.2) the function $F(\lambda)$ is absolutely continuous (in the weak sense), then we may replace the formulae (3.5) by the inverse Fourier transformation,

(c) in various models the reduced evolution operator is determined by means of the function $F(\lambda)$ (remember the Breit-Wigner formula and its modifications), then one is able to apply Theorem 4 directly.

Let us notice that alternative formulae to (3.5) of this paper could be deduced from Bochner theorems used to the similar aim by Sinha [5].

The second question which we shall discuss here concerns decay laws. Since they represent themselves directly measurable quantities (probability of nondecay at a given time), one can naturally ask whether it is possible to determine the energy spectrum from them. A knowledge of decay laws for sufficiently many states of the considered system gives to us the operator-valued function $V^{+}(t) V(t)$ cf. (2.3). Problem is now the following: does the function $V^{+}(t) V(t)$ determine the Hamiltonian or (what is the same) does it determine the function $V(t)$ ?

In the most simple case $\operatorname{dim} \mathscr{H}_{u}=1$ a reconstruction of the function $(\psi, V(t) \omega)$ from its modulus can be realized, however, some information about a behaviour of this function for complex values of $t$ is needed [7]. Generally, the problem is expressed by the equation

$$
V^{+}(t) V(t)=P(t)
$$


where $P(t)$ is a given operator-valued function with the following properties:

(i) the operator $P(t)$ is positive (and therefore Hermitian) for all $t \in \mathbb{R}$,

(ii) $P(0)=E_{u}$,

(iii) for any $t \in \mathbb{R}$ the inequality $\|P(t)\| \leqq 1$ holds,

(iv) $P(t)$ is weakly continuous with respect to $t$.

One can also assume $P(t)$ to obey some other (physically motivated) conditions. Notice that $P(t)$ need not be positive definite, if $\operatorname{dim} \mathscr{H}_{u}>1$ : in such a case the condition analogous to $(2.2 \mathrm{a})$ would have to hold

$$
V^{+}(t) V(t)=P(t)=P^{+}(-t)=\left[V^{+}(-t) V(-t)\right]^{+}=V(t) V^{+}(t),
$$

but $V(t)$ need not generally commute with $V^{+}(t)$.

Up to our knowledge, it is not known, under which assumptions there exists a WPO-function $V(t)$ solving the Equation (4.1). If such a solution exists, then it is not a unique one:

(a) a function $W(t) V(t), W(t)$ being any strongly continuous representation of one-parameter translation group on $\mathscr{H}_{w}$, solves the Equation (4.1) together with $V(t)$; if for example $\left[V\left(t_{1}\right), W\left(t_{2}\right)\right]=0$ for all $t_{1}, t_{2} \in \mathbb{R}$, then positive definiteness of $V(t)$ implies the same property for $W(t) V(t)$,

(b) if $\left[V^{+}(t), V(t)\right]=0$ for all $t>0$, then the function $V^{+}(t)$ solves the Equation (4.1) together with $V(t)$; if $V(t)$ is positive definite so is $V^{+}(t)$.

We do not know, whether (a) and (b) are all possible non-uniquenesses of solutions of the Equation (4.1) or not.

Concluding this part of discussion, a solution to "existence and uniqueness problem" of the Equation (4.1) is needed in order to decide whether a given operator-valued function $P(t)$ corresponds to some unstable system (i.e. represents a collection of decay laws) in the present formalism. Assuming now $P(t)$ to be such a function (to which a reduced evolution operator $V(t)$, and consequently a total Hamiltonian $H$ corresponds), we can easily see the following consequence of the non-uniqueness (a) (which is the more substantial one); for example an operator $H^{\prime}=H+G$ which obeys the conditions

(i) $G$ is self-adjoint,

(ii) $G$ is reduced by $\mathscr{H}_{u}$,

(iii) $G$ commutes with $H$,

can be taken by the same right as a Hamiltonian giving the function $P(t)$. Excluding the physically irrelevant possibility $G=\alpha I, \alpha \in \mathbb{R}$, the present example shows that a knowledge of $P(t)$ alone need not be sufficient in order to determine the Hamiltonian.

Acknowledgment. The author is indebted to Prof. V. Votruba and Dr. M. Havlíček for helpful and inspiring discussions.

\section{References}

1. Horwitz, L.P., Marchand, J.-P.: Helv. Phys. Acta 42, 801-807, 1039-1053 (1969)

2. Havlíček, M., Exner, P.: Czech. J. Phys. B23, 594-600 (1973)

3. Williams, D. N.: Commun. math. Phys. 21, 314-333 (1971)

4. Horwitz, L.P., LaVita, J., Marchand, J.-P.: J. Math. Phys. 12, 2537-2543 (1971) 
5. Sinha, K.: Helv. Phys. Acta 45, 619-628 (1972)

6. Lévy, M.: Nuovo Cimento 14, $612-624$ (1960)

7. Khalfin, L.A.: JETP 33, 1371-1382 (1957)

8. Exner, P.: Czech. J. Phys. B 26, $976-982$ (1976)

9. Sz.-Nagy, B., Foias, C.: Harmonic analysis of operators on Hilbert space. Amsterdam: NorthHolland Publ. Co. 1970

10. Akhiezer, N.I., Glazman,I.M.: Theory of linear operators in Hilbert space (Russian). Moscow: Nauka 1966

11. Sz.-Nagy, B.: Acta Sci. Math. Szeged 15, 87-92, 104-114 (1953)

12. Riesz, F., Sz.-Nagy, B.: Functional analysis. New York: Ungar Publ. Co. 1960

Blank, J., Exner, P., Havlíček, M.: Linear operators on Hilbert space I (Czech). Prague: SPN 1975

Communicated by H. Araki

Received August 25, 1975 